\title{
Uniform Signal Redundancy of Parasol and Midget Ganglion Cells in Primate Retina
}

\author{
Jeffrey L. Gauthier, ${ }^{1 \star}$ Greg D. Field, ${ }^{1 *}$ Alexander Sher, ${ }^{2}$ Jonathon Shlens, ${ }^{1,3}$ Martin Greschner, ${ }^{1}$ Alan M. Litke, ${ }^{2}$ \\ and E. J. Chichilnisky ${ }^{1}$ \\ ${ }^{1}$ Salk Institute for Biological Studies, La Jolla, California 92037, ${ }^{2}$ Santa Cruz Institute for Particle Physics, University of California, Santa Cruz, Santa Cruz, \\ California 95060, and ${ }^{3}$ University of California, Berkeley, Berkeley, California 94720
}

The collective representation of visual space in high resolution visual pathways was explored by simultaneously measuring the receptive fields of hundreds of $\mathrm{ON}$ and OFF midget and parasol ganglion cells in isolated primate retina. As expected, the receptive fields of all four cell types formed regular mosaics uniformly tiling the visual scene. Surprisingly, comparison of all four mosaics revealed that the overlap of neighboring receptive fields was nearly identical, for both the excitatory center and inhibitory surround components of the receptive field. These observations contrast sharply with the large differences in the dendritic overlap between the parasol and midget cell populations, revealing a surprising lack of correspondence between the anatomical and functional architecture in the dominant circuits of the primate retina.

\section{Introduction}

Populations of sensory neurons encode information collectively, and a fundamental aspect of the population code is signal redundancy: the degree to which features of sensory space are represented by more than one neuron. In the mammalian retina, $\sim 20$ types of retinal ganglion cells (RGCs) sample visual space (Dacey, 2004; Yamada et al., 2005), and each cell type forms a lattice of regularly spaced receptive fields (RFs) that overlap their neighbors to a greater or lesser degree (Peichl and Wässle, 1979; Devries and Baylor, 1997; Segev et al., 2006; van Wyk et al., 2006). Greater RF overlap implies greater signal redundancy in the neural encoding (Puchalla et al., 2005; Segev et al., 2006), which may be valuable for downstream computations that are sensitive to noise, but could also produce a less efficient neural representation. In the primate visual system, anatomical and physiological studies have yielded conflicting predictions about the degree of signal redundancy in the magnocellular and parvocellular pathways, which provide the highest-resolution visual signals to the brain and are a major focus of current research.

Received Nov. 3, 2008; revised Dec. 17, 2008; accepted Jan. 8, 2009.

This work was supported by National Institutes of Health (NIH) National Research Service Award 1 F31 NS054519-01 and Chapman Foundation (J.L.G.), the Helen Hay Whitney Foundation (G.D.F.), Burroughs Wellcome Fund Career Award at Scientific Interface (A.S.), Deutscher Akademischer Austausch Dienst and Deutsche Forschungsgemeinschaft (M.G.), National Science Foundation (NSF) Integrative Graduate Education and Research Traineeship DGE-033451, Miller Institute for Basic Science Research (J.S.), McKnight Foundation (A.M.L., E.J.C.), NSF Grant PHY-0417175 (A.M.L.), and NIH Grant EY017992 (E.J.C.). We thank B. Kutka and C. Hulse for technical assistance; M. I. Grivich, D. Petrusca, W. Dabrowski, A. Grillo, P. Grybos, P. Hottowy, and S. Kachiguine for technical development; H. Fox, M. Taffe, E. Callaway, and K. Osborn for providing access to retinas; S. Barry for machining; D. Marshak for helpful discussions; and E. Callaway and M. Feller for comments on this manuscript. This research was supported in part by the National Science Foundation through TeraGrid resources provided by the San Diego Supercomputer Center (Cooperative Agreements 05253071 and 0438741).

*J.L.G. and G.D.F. contributed equally to this work.

Correspondence should be addressed to E.J. Chichilnisky, Systems Neurobiology, The Salk Institute, 10010 North Torrey Pines Road, La Jolla, CA 92037. E-mail: ej@salk.edu.

DOI:10.1523/JNEUROSCI.5294-08.2009

Copyright $\odot 2009$ Society for Neuroscience $\quad$ 0270-6474/09/294675-06\$15.00/0
Anatomical findings suggest a substantial difference in the signal redundancy of parasol and midget retinal ganglion cells (Polyak, 1941; Watanabe and Rodieck, 1989), which form the dominant input to the magnocellular and parvocellular pathways, respectively (Leventhal et al., 1981; Perry and Cowey, 1981; Perry et al., 1984; Dacey and Brace, 1992). The dendritic fields (DFs) of parasol cells overlap extensively, while midget cell DFs exhibit no overlap (Dacey and Brace, 1992; Dacey and Petersen, 1992; Dacey, 1993), as depicted in Figure $1 A$. If the RFs of these populations exhibited a correspondingly large difference in overlap, parasol cells would sample the visual scene with high signal redundancy, while midget cells would provide more independent samples, perhaps reflecting their distinct roles in visual function (Livingstone and Hubel, 1988).

A conflicting prediction arises from physiological measurements in rabbit retina. One study (DeVries and Baylor, 1997) revealed nearly identical RF overlap in a variety of different ganglion cell types (see also Borghuis et al., 2008) (but see van Wyk et al., 2006). This finding suggests that a single degree of signal redundancy can satisfy a range of visual processing requirements. In principle, parasol and midget cells could also exhibit a common degree of signal redundancy, despite their substantial anatomical differences and different roles in vision. Although the RF structure of individual parasol and midget cells has been examined previously (Croner and Kaplan, 1995) (see also Lee, 1996), the RF overlap of these important RGC populations has not.

We examined the collective representation of visual space in populations of parasol and midget ganglion cells by applying large-scale electrophysiological recordings to isolated peripheral primate retina (Litke et al., 2004; Frechette et al., 2005). Within the regular mosaic formed by each cell type, ON and OFF midget and parasol cells exhibited nearly identical RF overlap, in both the center and surround components of the RF. Thus, retinal circuitry precisely compensates for striking differences in morphol- 
ogy, producing a common functional organization in the parvocellular and magnocellular pathways.

\section{Materials and Methods}

Preparation and recording. Retinas were obtained and recorded as described previously (Chichilnisky and Kalmar, 2002; Field et al., 2007). Briefly, eyes were enucleated from terminally anesthetized macaque monkeys (Macaca mulatta, Macaca fascicularis) from several sources (Field et al., 2007) in accordance with institutional guidelines for the care and use of animals. Immediately after enucleation, the anterior portion of the eye and vitreous were removed in room light. Following a dark incubation period, patches of peripheral retina were isolated from the pigment epithelium and placed flat, RGC side down, on a planar array of 512 extracellular microelectrodes covering an area $1890 \times 900 \mu \mathrm{m}$. During recording, the retina was kept at $33-35^{\circ} \mathrm{C}$ and was perfused with Ames' solution bubbled with $95 \% \mathrm{O}_{2}$ and $5 \% \mathrm{CO}_{2}, \mathrm{pH}$ 7.4.

Raw voltage recordings were analyzed off-line to isolate spikes from single cells, as described previously (Field et al., 2007). Briefly, candidate spikes were detected using a threshold on each electrode, and the voltage waveform on the reference and nearby electrodes was saved. Spikes were clustered based on waveform shape, and clusters were identified as candidate neurons if they exhibited a refractory period and an average spike rate $>1 \mathrm{~Hz}$. Duplicate recordings of the same cell were identified by temporal cross-correlation and removed.

RF characterization. RFs were mapped as described previously (Chichilnisky and Kalmar, 2002; Field et al., 2007). Briefly, the optically reduced image of a gamma-corrected cathode ray tube computer display (Sony Multiscan E100) refreshing at $120 \mathrm{~Hz}$ was focused on the photoreceptor outer segments, and low photopic intensity was achieved using neutral density filters in the light path. A white noise stimulus was presented, consisting of a lattice of squares (pixels), each flickering randomly and independently at 30 or $120 \mathrm{~Hz}$ (Chichilnisky, 2001), with the intensities of the red, green, and blue display phosphors varying independently. The contrast of this stimulus for each of the three display phosphors was 96\% (SD of modulation divided by mean intensity), and the side lengths of individual pixels varied from 30 to $60 \mu \mathrm{m}$. The RF of each recorded cell was mapped by computing the spike-triggered average (STA) stimulus during the white noise presentation (Rieke et al., 1997; Chichilnisky, 2001).

RFs were summarized by fitting with a parametric model. The model consisted of the product of three profiles: spatial, temporal, and chromatic (Chichilnisky and Kalmar, 2002). The temporal profile was a difference of low-pass filters. The spatial profile consisted of a difference of two-dimensional elliptical Gaussian functions. The chromatic profile was the relative weighting of the three monitor phosphors. Surrounds were relatively weak, so could not be fitted individually in a robust manner. However, on average, the STA spatial profile was well described by a fit in which the radius of the surround was twice that of the center. Thus, the following procedure was used to fit the spatial profile. A single twodimensional Gaussian was fitted to the STA of each cell, generating a rough estimate of receptive field (RF) center location and radius. An inhibitory surround was then introduced, and the fit was reoptimized over all remaining parameters, with the surround radius constrained to be twice the center radius. Several parameters of the fit were extracted to visualize RF extent: the location of the Gaussian fit center, the SDs along the major and minor axes, and the angle of the major axis. These parameters defined an ellipse for each cell that represented the 1 SD contour of the Gaussian fit [that is, the contour level at which the sensitivity profile achieved a value of $\exp (-1 / 2)$ relative to its peak]. In figures, RF outlines are represented using this contour.

Cell type classification and identification. The morphological type of recorded cells was determined using a two-step procedure, as described previously (Chichilnisky and Kalmar, 2002; Field et al., 2007). Briefly, cells were first grouped into functional cell classes based on their light response properties. Correspondences between functional classes and morphological types were determined by density and light response properties. This procedure definitively identified the ON and OFF parasol and midget cells in each recording.

Coverage. Anatomical coverage of dendritic fields is usually defined as the average number of cells sampling any given point in visual space. In the case of RFs approximated by Gaussian fits, the RF extent is not well defined, so overlap was analyzed using the normalized nearest neighbor distance (NNND). For a given cell, the NNND is given by $2 R /\left(\sigma_{1}+\sigma_{2}\right)$, where $R$ is the distance between the Gaussian fit center points of each cell and its nearest neighbor in the mosaic, and $\sigma_{1}$ and $\sigma_{2}$ are SDs of the fits measured along the line connecting the center points. Thus, for nearest neighbors that just touch at the $1 \mathrm{SD}$ contour, the NNND is 2. Note that the NNND is large when overlap is small, and vice versa. This representation has the advantages of being closely related to a standard anatomical measure (NND) and allowing for unbiased comparison of overlap in cell types with different absolute sizes.

A control analysis verified that the estimate of the NNND was not affected by different pixel sizes. In one preparation, RFs were measured using several pixel sizes (96, 60, and $18 \mu \mathrm{m}$ per pixel), and the NNND was computed for the ON and OFF parasol cells and ON midget cells. Within each cell type, the modal NNND value varied by $<12 \%$ across the three pixel sizes, demonstrating that pixel size did not significantly affect the NNND.

RF profiles. The average RF profile of neighboring cells was computed separately for each mosaic in several steps. First, the spatial part of the RF was obtained by applying singular value decomposition to STA frames in which the contrast intensity was at least $20 \%$ of the peak contrast (usually $30-80 \mathrm{~ms}$ before the spike). Second, the center point of each spatial RF was estimated by taking the center of mass of all pixels with amplitude at least half that of the highest amplitude pixel. Third, the amplitude of each STA was scaled so that the central region of the STA had unit variance. The central region was the circle centered on the center of mass with a radius of 3 times the nearest neighbor spacing. Fourth, the line connecting each cell to its nearest neighbor was computed. Along this line, absolute distance was normalized so that the nearest neighbor was exactly 1 unit away. Fifth, the RF amplitude of the reference cell and its nearest neighbor at each pixel along the nearest neighbor line were extracted. Thus the RF intensity was a continuously valued function with a staircase shape representing the distinct pixels in the RF. Finally, the RF profiles were averaged for all reference cells. The amplitude was normalized so that the variance over the range shown in Figure 3 had unit variance.

\section{Results}

\section{Receptive field mosaics of parasol and midget cells}

Light responses of hundreds of retinal ganglion cells were simultaneously recorded in isolated segments of macaque monkey retina. The receptive field ( $\mathrm{RF}$ ) of each cell was identified using reverse correlation with a white noise stimulus (Chichilnisky and Kalmar, 2002). Cells were identified as ON and OFF parasol and midget based on their light response properties and density (Field et al., 2007). To visualize RFs, the center component was extracted from a difference of Gaussians fit to the spatial sensitivity profile (see Materials and Methods) (Rodieck, 1965). The RF center was represented graphically by the $1 \mathrm{SD}$ contour of the Gaussian fit (Devries and Baylor, 1997). This representation is arbitrary and does not reveal the full extent of the RF, however, it permits a meaningful comparison of RFs of different cell types. Figure $1, B$ and $C$, shows the RF centers of simultaneously recorded $\mathrm{ON}$ and OFF parasol and midget cells from two preparations. As expected from previous work (Dacey and Brace, 1992; Dacey, 1993; Chichilnisky and Kalmar, 2002; Frechette et al., 2005; Field et al., 2007), the RFs of each cell type formed a regularly spaced lattice, or mosaic. The complete mosaic structure indicates that, over some regions of retina, all or nearly all cells of each type were recorded. This complete sampling is essential for reliably measuring the signal redundancy in each cell type.

\section{Overlap of neighboring receptive field centers}

Visual inspection of Figure 1, $B$ and $C$, suggests that parasol and midget RFs exhibit similar RF overlap: the RFs of all four cell types abut their neighbors at approximately the $1 \mathrm{SD}$ contour 
A

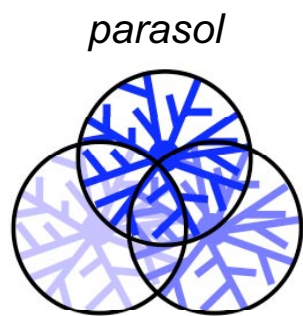

B

ON
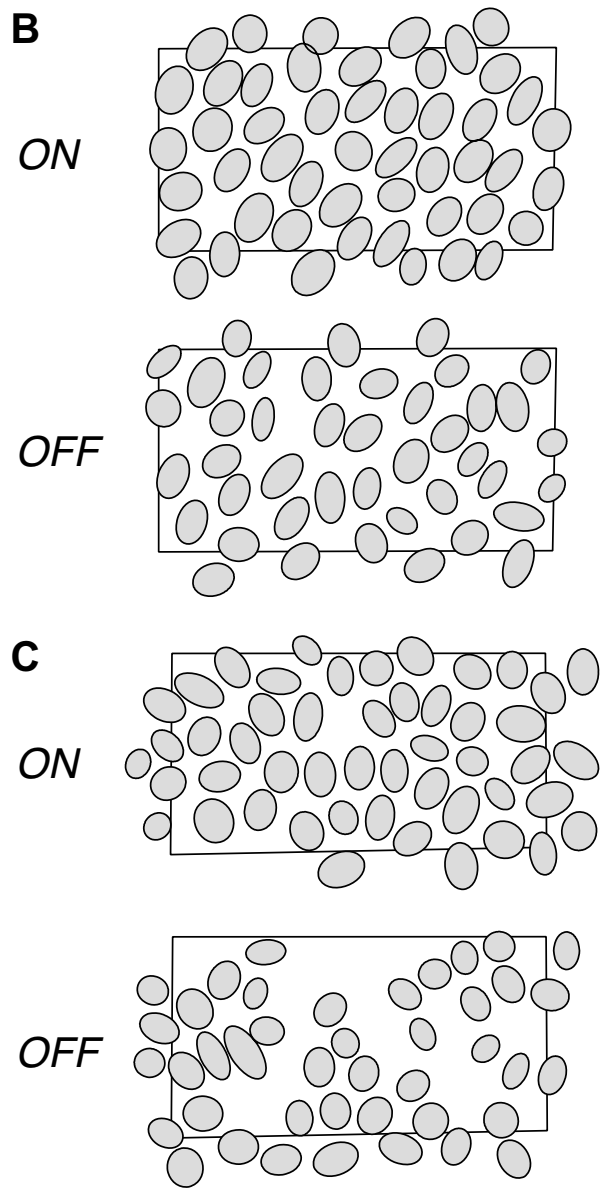

midget
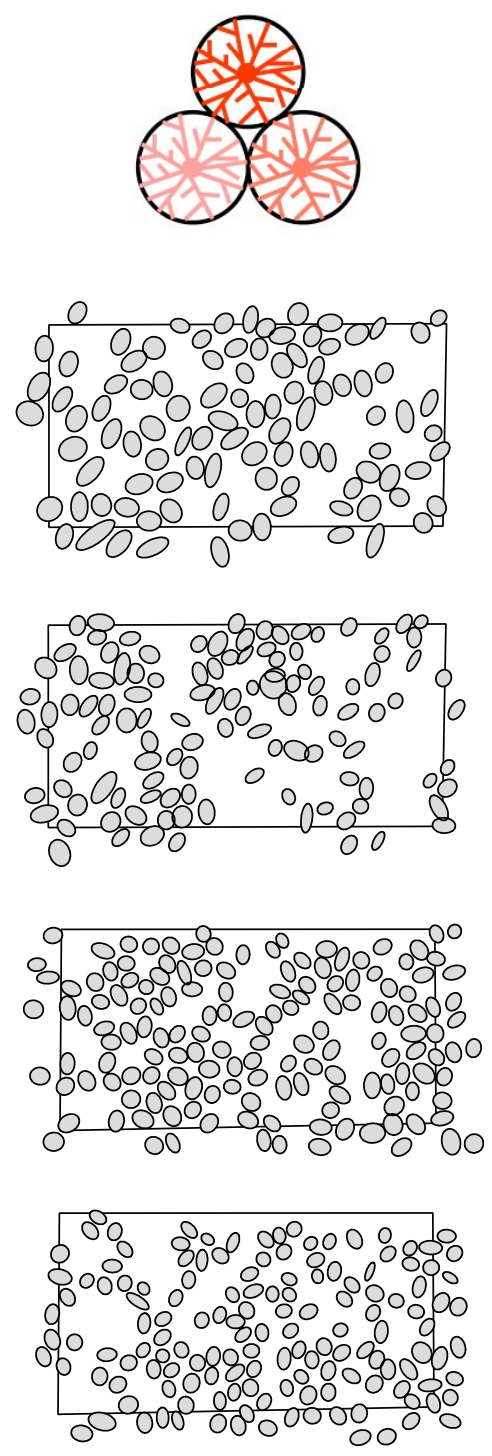

Figure 1. Parasol and midget RF mosaics and anatomical prediction. $A$, Previous anatomical findings indicate that parasol cell dendritic fields overlap substantially, with the tips of each dendritic field reaching the soma of its neighbors in the mosaic, while midget cell dendritic fields abut at their boundaries. $\boldsymbol{B}$, Each panel shows the RFs of simultaneously recorded ON and OFF parasol and midget cells from one retina, with each RF represented as the 1 SD contour of a Gaussian fit to the RF center. Note that this does not represent the full extent of the RF (see text for details). Black rectangles indicate the outline of the recording array. Gaps in the mosaic probably represent unrecorded cells. Retinal temporal equivalent eccentricity: $6.4 \mathrm{~mm}$. $\boldsymbol{C}$, Same as in $\boldsymbol{B}$ for a second preparation; temporal equivalent eccentricity $9.0 \mathrm{~mm}$.

shown by the outlines. Note that this observation does not imply no RF overlap: a substantial fraction of the RF lies outside the 1 $\mathrm{SD}$ contour. However, the similar pattern across cell types suggests equal overlap.

This suggestion was confirmed quantitatively by measuring the spacing of cells in each mosaic, relative to the size of the RFs. The distance between neighboring RF centers was divided by the equivalent RF radius, producing a normalized nearest neighbor distance (NNND; see Materials and Methods) (Devries and Baylor, 1997). For a mosaic with high (low) overlap, the NNND will be small (large). When neighboring RFs just touch at the $1 \mathrm{SD}$ contour, the NNND value is 2 . Figure $2 A$ shows the NNNDs for each cell type from the preparation of Figure $1 B$. The modal NNND of each cell type is represented graphically beneath the abscissa. For all four cell types, the modal NNND was near 2, confirming the impression that the RFs of neighboring cells abut approximately at the 1 SD contour. Figure $2 B$ shows similar results for the preparation of Figure $1 C$.

Parasol and midget cells exhibited nearly identical RF overlap in multiple recordings over a range of eccentricities. Figure $2 C$ shows modal NNNDs of parasol and midget cells, summarizing the RF overlap of 36 mosaics (3221 cells, 10 preparations). ON and OFF populations are represented by open and closed circles, respectively. The data fall near the identity line (solid line) that indicates equal RF overlap. This finding contrasts with the approximately twofold difference predicted from DF overlap (dashed line). On average, ON cells exhibited slightly more overlap than OFF cells, for both parasol and midget populations (Chichilnisky and Kalmar, 2002). Figure $2 D$ reveals that overlap did not vary with retinal eccentricity across the peripheral visual field. Over the range of eccentricities recorded, $\mathrm{RF}$ sizes varied by approximately twofold. Thus, the observed overlap is a consistent feature that is independent of absolute RF size.

\section{Overlap of neighboring receptive field profiles}

The above results were confirmed over the entire extent of the RF, including the inhibitory surround, by directly examining light sensitivity profiles. For each reference cell, light sensitivity was measured along the line connecting the RF center to that of the nearest neighbor in the mosaic, producing two spatial profiles: one for the reference cell, and one for the neighbor. The reference and neighbor profiles were averaged across every reference cell in the mosaic. These profiles were normalized to focus on profile shape independently of absolute size, spacing, and sensitivity (see Materials and Methods).

Figure $3 A$ shows the resulting average neighbor profiles for $\mathrm{ON}$ and OFF parasol and midget cells from the preparation of Figure $1 C$. All four cell types exhibited nearly identical overlap in neighbor profiles, though the ON parasol cells exhibited a slightly flatter peak, and, as in Figure 2C, ON cells exhibited slightly greater overlap. The closely overlaying profiles confirm that RF overlap is nearly equal in all four cell types. Unlike the parametric analysis above, this analysis also shows the contribution of the inhibitory RF surrounds, which overlaid closely. Similar results were observed in a second preparation (Fig. 3B).

\section{Discussion}

$\mathrm{ON}$ and OFF parasol and midget cells exhibited nearly identical RF profiles and overlap in the conditions examined. Thus the 
A

parasol

midget
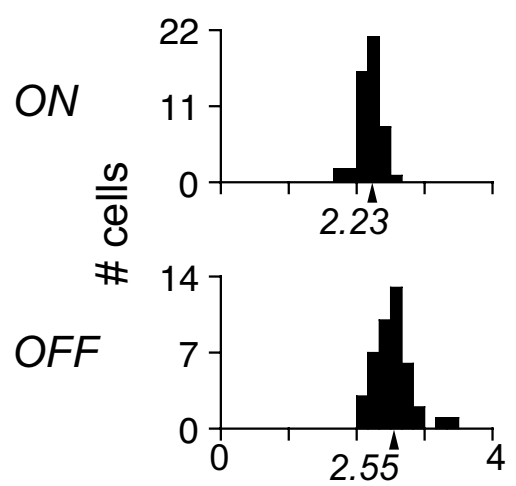
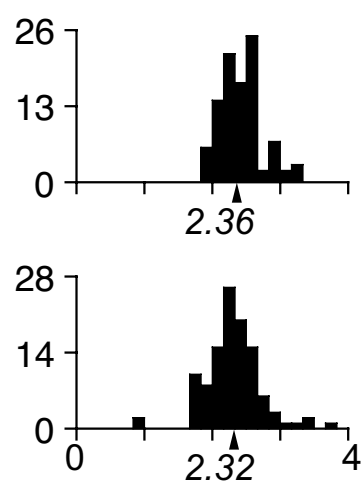

B

B parasol
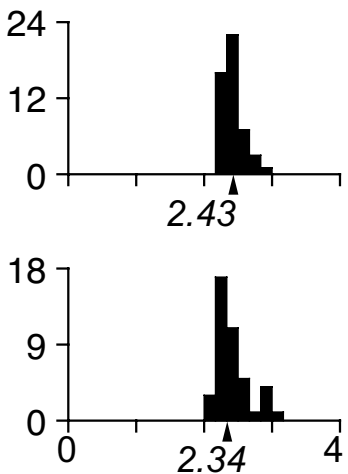

midget
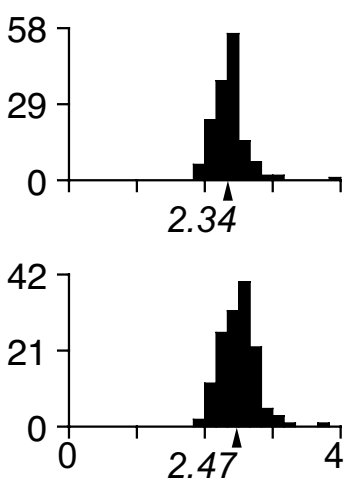

normalized nearest neighbor distance (NNND)
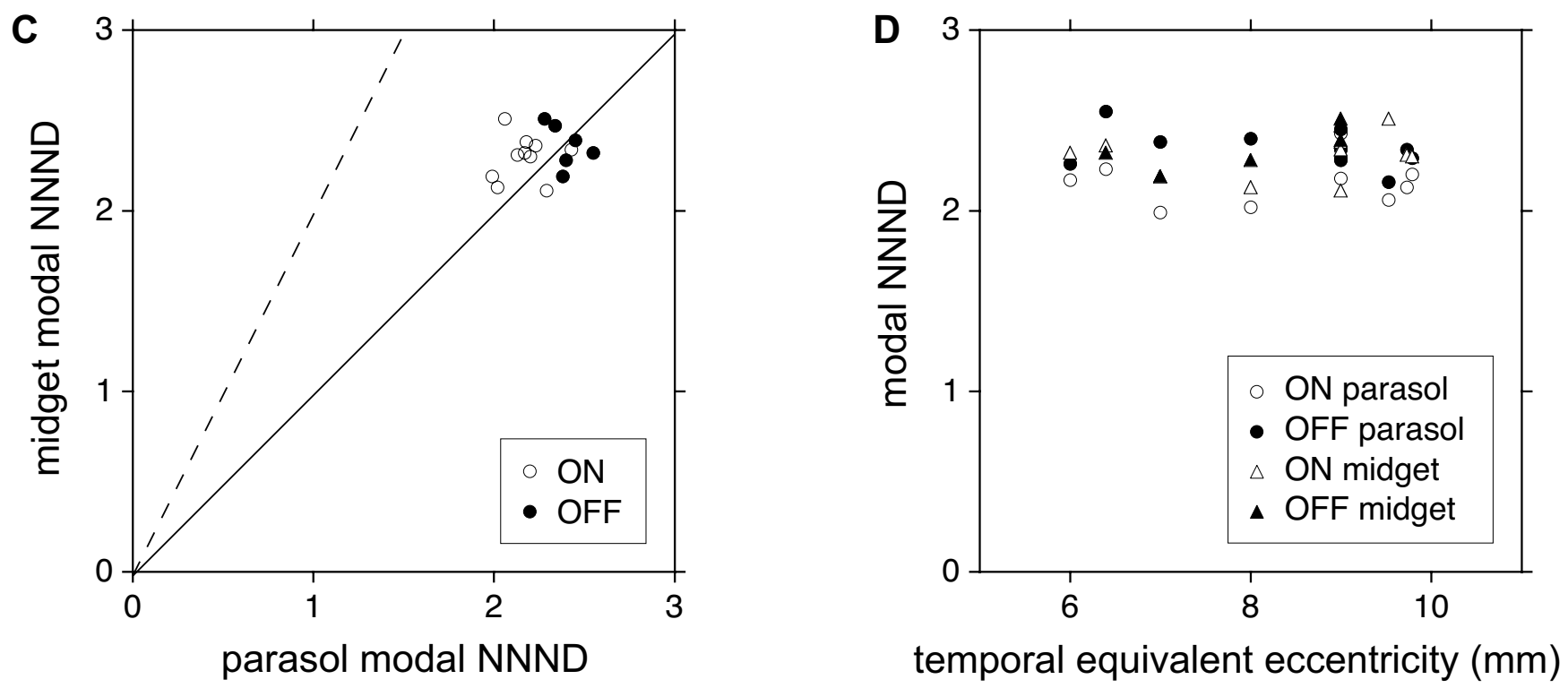

Figure 2. Quantitative analysis of RF overlap. The normalized nearest neighbor distance (NNND) expresses RF spacing relative to RF size; if two mosaics have the same degree of RF overlap, they will have the same NNND. $A$, NNND values for the mosaics of parasol and midget cells shown in Figure $1 B$, with the modal NNND indicated on the abscissa. Because the recordings did not sample every cell in the mosaic, the modal NNND was computed using the mean of the densest $75 \%$ of values, which excluded outlying points. The robustness of this calculation was confirmed by subsampling analysis (see Materials and Methods). B, Similar data, for the preparation in Figure 1C. C, Data summarizing the RF overlap of 36 mosaics. Modal NNND values of simultaneously recorded parasol and midget mosaics are compared separately for ON cells (open circles) and OFF cells (filled circles). Solid line indicates equality, dashed line indicates the prediction from anatomical findings that NNND should be approximately twice as large for midget cells. D, NNND values as a function of retinal eccentricity, for ON (open) and OFF (filled) cells of both midget (triangle) and parasol (circle) types.

retinal circuitry precisely counteracts substantial differences in the dendritic overlap in these populations, producing a highly uniform functional organization in the magnocellular and parvocellular visual pathways.

\section{Functional versus anatomical overlap}

The striking discrepancy between structure and function distinguishes the present study from previous work on RGC populations for which less anatomical information is available (Devries and Baylor, 1997; Segev et al., 2006; van Wyk et al., 2006; Borghuis et al., 2008). Parasol cell DFs overlap substantially, while midget cell DFs overlap little or not at all (Dacey and Brace, 1992; Dacey and Petersen, 1992; Dacey, 1993) (Fig. 1A). Therefore, the present results imply that the relationship between RF and DF is not universal, but is unique to each cell type. This fact presumably reflects the diversity of the bipolar and amacrine cell circuits contacting each RGC type (Field and Chichilnisky, 2007), an is- sue that has been raised but not resolved in previous studies of the relation between RF and DF structure (Peichl and Wässle, 1981, 1983; Yang and Masland, 1994; Brown et al., 2000). For example, the relatively larger size of bipolar cell RFs in the midget pathway (Dacey et al., 2000) could help explain the similarity of overlap in the midget and parasol populations.

Signal redundancy in parallel visual pathways

Previous work presents a complex picture of RF overlap and signal redundancy in different RGC types. The first direct measurement of RF overlap revealed striking homogeneity among several RGC types in the rabbit retina (Devries and Baylor, 1997), and provided a theoretical suggestion that this degree of overlap could be optimal for many RGC types (see also Borghuis et al., 2008). However, several subsequent studies questioned this conclusion by demonstrating that RF overlap varies significantly across different cell types. One study (van Wyk et al., 2006) 

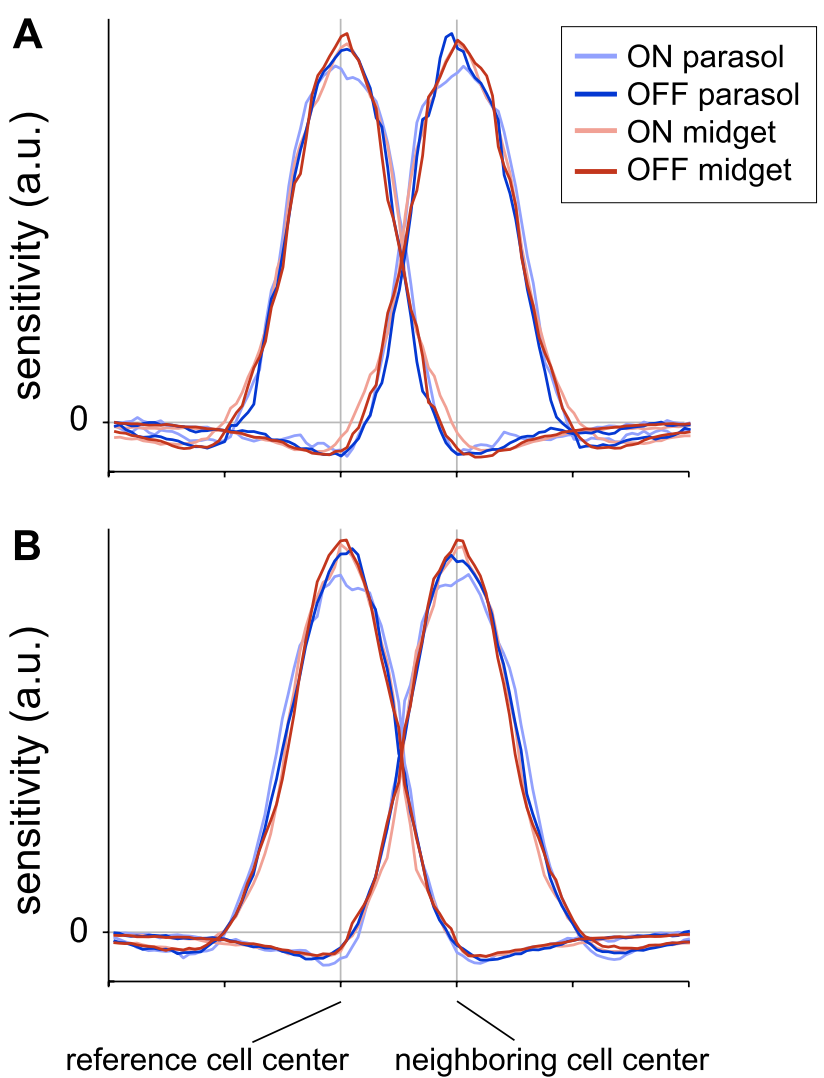

\section{normalized distance}

Figure 3. Nearest neighbor RF profiles for ON and OFF parasol and midget cells. A, For each cell type, the average RF profiles of a cell and its nearest neighbor were computed directly by interpolation of the spatial receptive field (see Materials and Methods). Distance and amplitude were normalized to focus on the RF profile shape independently of absolute size, spacing, and sensitivity. Distance between centers (vertical bars) was normalized for each pair of nearest neighbors (see Materials and Methods). Sensitivity was normalized to have the same variance across cell types (see Materials and Methods). Data are from the preparation shown in Figure $1 C$. $\boldsymbol{B}$, Same as $\boldsymbol{A}$, from a second preparation (temporal equivalent eccentricity $9 \mathrm{~mm}$ ).

showed that the densest cell type of the rabbit retina exhibits much higher RF overlap than originally reported (Devries and Baylor, 1997). Another recent study in primate retina showed that ON parasol cells have slightly higher RF overlap than small bistratified cells (Field et al., 2007), which exhibit a distinctive bistratified dendritic morphology and color-opponent light responses. In the larval tiger salamander, RF overlap seems to vary for different RGC types (Segev et al., 2006), though cell type classification in this species is less certain. Theoretical work has also suggested that the optimal degree of RF overlap can depend on factors unique to each cell type (Vincent and Baddeley, 2003; Vincent et al., 2005). Finally, no previous studies have systematically characterized the shape of center and surround profiles in simultaneously recorded populations of RGCs.

While the present results do not provide a unified view of how or why signal redundancy varies across cell types, they unambiguously demonstrate nearly identical redundancy in the dominant visual pathways of primate retina, both in the center and surround of the RF. This result is somewhat surprising in light of the distinct functional roles of the two pathways. Neurons in the magnocellular and parvocellular layers of the lateral geniculate nucleus (LGN), which receive predominantly parasol and midget inputs, respectively (Leventhal et al., 1981; Perry and Cowey,
1981; Perry et al., 1984; Dacey and Brace, 1992), exhibit very different projections to visual cortex and systematically different response properties (Merigan and Maunsell, 1993; Callaway, 2005). The visual signals carried by these two pathways are thought to mediate largely distinct visual functions, such as perception of change and motion, or fine spatial detail and color, respectively (Livingstone and Hubel, 1988). The unique functions of these pathways begin to emerge in the distinct light response properties of midget and parasol cells (Lee et al., 1989a,b; De Monasterio and Gouras, 1975; Kaplan and Shapley, 1986; Benardete et al., 1992; Troy and Lee, 1994; Croner and Kaplan, 1995). In principle, the different functions of the magnocellular and parvocellular pathways could demand different degrees of signal redundancy in the underlying RGC signals. Instead, the striking similarity suggests that the two high-density pathways may share a common functional requirement, such as the need to efficiently encode visual information in a limited number of optic nerve fibers, and that the observed redundancy may optimally satisfy this requirement.

\section{Future}

An important caveat to the present findings is that RF overlap could vary with light level. All the present data were gathered at low photopic light levels (cone-dominated signaling). Different results could hold at scotopic light levels [rod-dominated signaling (Barlow et al., 1957)], because of the distinct circuitry that conveys rod signals to RGCs (Kolb and Famiglietti, 1974; Bloomfield and Dacheux, 2001), and because of the distinct functional requirements in conditions where the visual signal is limited by quantum fluctuations in photon absorption (Field et al., 2005).

A perplexing problem is how developmental mechanisms produce homogeneous functional organization in the parasol and midget cells despite their structural differences. Clearly, the mechanisms responsible for dendrite growth must operate differently in the two populations. The fact that these mechanisms are precisely counterbalanced with other elements of retinal circuitry, producing nearly identical RF overlap and profiles, suggests that development of RGC mosaics may be governed by the functional outcome, and thus may rely partly on visual experience (Feller and Scanziani, 2005; Hooks and Chen, 2007; White and Fitzpatrick, 2007).

\section{References}

Barlow HB, Fitzhugh R, Kuffler SW (1957) Change of organization in the receptive fields of the cat's retina during dark adaptation. J Physiol 137:338-354.

Benardete EA, Kaplan E, Knight BW (1992) Contrast gain control in the primate retina: $\mathrm{P}$ cells are not $\mathrm{X}$-like, some $\mathrm{M}$ cells are. Vis Neurosci 8:483-486.

Bloomfield SA, Dacheux RF (2001) Rod vision: pathways and processing in the mammalian retina. Prog Retin Eye Res 20:351-384.

Borghuis BG, Ratliff CP, Smith RG, Sterling P, Balasubramanian V (2008) Design of a neuronal array. J Neurosci 28:3178-3189.

Brown SP, He S, Masland RH (2000) Receptive field microstructure and dendritic geometry of retinal ganglion cells. Neuron 27:371-383.

Callaway EM (2005) Structure and function of parallel pathways in the primate early visual system. J Physiol 566:13-19.

Chichilnisky EJ (2001) A simple white noise analysis of neuronal light responses. Network 12:199-213.

Chichilnisky EJ, Kalmar RS (2002) Functional asymmetries in ON and OFF ganglion cells of primate retina. J Neurosci 22:2737-2747.

Croner LJ, Kaplan E (1995) Receptive fields of P and M ganglion cells across the primate retina. Vision Res 35:7-24.

Dacey DM (1993) The mosaic of midget ganglion cells in the human retina. J Neurosci 13:5334-5355.

Dacey DM (2004) Origins of perception: retinal ganglion cell diversity and 
the creation of parallel visual pathways. In: The cognitive neurosciences (Gazzaniga MS, ed), pp 281-301. Cambridge, MA: MIT.

Dacey DM, Brace S (1992) A coupled network for parasol but not midget ganglion cells in the primate retina. Vis Neurosci 9:279-290.

Dacey DM, Petersen MR (1992) Dendritic field size and morphology of midget and parasol ganglion cells of the human retina. Proc Natl Acad Sci U S A 89:9666-9670.

Dacey D, Packer OS, Diller L, Brainard D, Peterson B, Lee B (2000) Center surround receptive field structure of cone bipolar cells in primate retina. Vision Res 40:1801-1811.

De Monasterio FM, Gouras P (1975) Functional properties of ganglion cells of the rhesus monkey retina. J Physiol 251:167-195.

Devries SH, Baylor DA (1997) Mosaic arrangement of ganglion cell receptive fields in rabbit retina. J Neurophysiol 78:2048-2060.

Feller MB, Scanziani M (2005) A precritical period for plasticity in visual cortex. Curr Opin Neurobiol 15:94-100.

Field GD, Chichilnisky EJ (2007) Information processing in the primate retina: circuitry and coding. Annu Rev Neurosci 30:1-30.

Field GD, Sampath AP, Rieke F (2005) Retinal processing near absolute threshold: from behavior to mechanism. Annu Rev Physiol 67:491-514.

Field GD, Sher A, Gauthier JL, Greschner M, Shlens J, Litke AM, Chichilnisky EJ (2007) Spatial properties and functional organization of small bistratified ganglion cells in primate retina. J Neurosci 27:13261-13272.

Frechette ES, Sher A, Grivich MI, Petrusca D, Litke AM, Chichilnisky EJ (2005) Fidelity of the ensemble code for visual motion in primate retina. J Neurophysiol 94:119-135.

Hooks BM, Chen C (2007) Critical periods in the visual system: changing views for a model of experience-dependent plasticity. Neuron 56:312-326.

Kaplan E, Shapley RM (1986) The primate retina contains two types of ganglion cells, with high and low contrast sensitivity. Proc Natl Acad Sci U S A 83:2755-2757.

Kolb H, Famiglietti EV (1974) Rod and cone pathways in the inner plexiform layer of cat retina. Science 186:47-49.

Lee BB (1996) Receptive field structure in the primate retina. Vision Res 36:631-644.

Lee BB, Martin PR, Valberg A (1989a) Sensitivity of macaque retinal ganglion cells to chromatic and luminance flicker. J Physiol 414:223-243.

Lee BB, Martin PR, Valberg A (1989b) Amplitude and phase of responses of macaque retinal ganglion cells to flickering stimuli. J Physiol 414:245-263.

Leventhal AG, Rodieck RW, Dreher B (1981) Retinal ganglion cell classes in the Old World monkey: morphology and central projections. Science 213:1139-1142.

Litke AM, Bezayiff N, Chichilnisky EJ, Cunningham W, Dabrowski W, Grillo AA, Grivich M, Grybos P, Hottowy P, Kachiguine S, Kalmar RS, Mathie- son K, Petrusca D, Rahman M, Sher A (2004) What does the eye tell the brain? Development of a system for the large-scale recording of retinal output activity. IEEE Trans Nucl Sci 51:1434-1440.

Livingstone M, Hubel D (1988) Segregation of form, color, movement, and depth: anatomy, physiology, and perception. Science 240:740-749.

Merigan WH, Maunsell JH (1993) How parallel are the primate visual pathways? Annu Rev Neurosci 16:369-402.

Peichl L, Wässle H (1979) Size, scatter and coverage of ganglion cell receptive field centres in the cat retina. J Physiol 291:117-141.

Peichl L, Wässle H (1981) Morphological identification of on- and offcentre brisk transient $(\mathrm{Y})$ cells in the cat retina. Proc R Soc Lond B Biol Sci 212:139-153.

Peichl L, Wässle H (1983) The structural correlate of the receptive field centre of alpha ganglion cells in the cat retina. J Physiol 341:309-324.

Perry VH, Cowey A (1981) The morphological correlates of X- and Y-like retinal ganglion cells in the retina of monkeys. Exp Brain Res 43:226-228.

Perry VH, Oehler R, Cowey A (1984) Retinal ganglion cells that project to the dorsal lateral geniculate nucleus in the macaque monkey. Neuroscience 12:1101-1123.

Polyak SL (1941) The retina. Chicago: University of Chicago.

Puchalla JL, Schneidman E, Harris RA, Berry MJ (2005) Redundancy in the population code of the retina. Neuron 46:493-504.

Rieke F, Warland D, Steveninck RR, Bialek W (1997) Spikes: exploring the neural code. Cambridge, MA: MIT.

Rodieck RW (1965) Quantitative analysis of cat retinal ganglion cell response to visual stimuli. Vision Res 5:583-601.

Segev R, Puchalla J, Berry MJ 2nd (2006) Functional organization of ganglion cells in the salamander retina. J Neurophysiol 95:2277-2292.

Troy JB, Lee BB (1994) Steady discharges of macaque retinal ganglion cells. Vis Neurosci 11:111-118.

van Wyk M, Taylor WR, Vaney DI (2006) Local edge detectors: a substrate for fine spatial vision at low temporal frequencies in rabbit retina. J Neurosci 26:13250-13263.

Vincent BT, Baddeley RJ (2003) Synaptic energy efficiency in retinal processing. Vision Res 43:1283-1290.

Vincent BT, Baddeley RJ, Troscianko T, Gilchrist ID (2005) Is the early visual system optimised to be energy efficient? Network 16:175-190.

Watanabe M, Rodieck RW (1989) Parasol and midget ganglion cells of the primate retina. J Comp Neurol 289:434-454.

White LE, Fitzpatrick D (2007) Vision and cortical map development. Neuron 56:327-338.

Yamada ES, Bordt AS, Marshak DW (2005) Wide-field ganglion cells in macaque retinas. Vis Neurosci 22:383-393.

Yang G, Masland RH (1994) Receptive fields and dendritic structure of directionally selective retinal ganglion cells. J Neurosci 14:5267-5280. 